\title{
Correction to: Do people exploit risk-reward structures to simplify information processing in risky choice?
}

\section{Christina Leuker $^{1}$ D $\cdot$ Thorsten Pachur $^{1} \cdot$ Ralph Hertwig $^{1} \cdot$ Timothy J. Pleskac $^{1,2}$}

Published online: 1 August 2019

(C) The Author(s) 2019

\section{Correction to: Journal of the Economic Science Association https://doi.org/10.1007/s40881-019-00068-y}

In the original publication of the article, the author's correction was missed in Table 1. The original article has been corrected and the correct Table 1 is given below.

The original article can be found online at https://doi.org/10.1007/s40881-019-00068-y.

\section{Christina Leuker}

leuker@mpib-berlin.mpg.de

1 Max Planck Institute for Human Development, Berlin, Germany

2 University of Kansas Max Planck Institute for Human Development, Berlin, Germany 
Table 1 Overview of regression models for processing and choice. Reference group set for environment: "uncorrelated". Models included a random effect for "participant." Coefficients are the mean and the 95\% credible intervals of the posterior distributions. Credible differences in bold

\begin{tabular}{|c|c|c|}
\hline \multirow[t]{2}{*}{ Regression } & \multicolumn{2}{|c|}{ Condition (Within-participant) } \\
\hline & Best & Fast \\
\hline \multicolumn{3}{|l|}{ Choice: Higher EV (1) } \\
\hline (Intercept) & $0.61[0.43,0.79]$ & $0.14[-0.01,0.29]$ \\
\hline EV difference & $0.13[0.12,0.14]$ & $0.06[0.05,0.07]$ \\
\hline Environment (Negative) & $-0.35[-0.60,-0.11]$ & $-0.23[-0.42,-0.03]$ \\
\hline Environment (Positive) & $-0.30[-0.54,-0.05]$ & $-0.18[-0.38,0.01]$ \\
\hline \multicolumn{3}{|l|}{ Processing: RTs (2) } \\
\hline (Intercept) & $4.03[3.50,4.57]$ & $1.01[0.95,1.06]$ \\
\hline EV difference & $-0.02[-0.03,-0.14]$ & $0.00[0.00,0.00]$ \\
\hline Environment (Negative) & $-1.10[-1.86,-0.34]$ & $-0.04[-0.11,0.03]$ \\
\hline Environment (Positive) & $-0.63[-1.38,0.13]$ & $-0.02[-0.10,0.05]$ \\
\hline \multicolumn{3}{|l|}{ Processing: AOIs (3) } \\
\hline (Intercept) & $3.18[2.94,3.41]$ & $2.09[1.82,2.36]$ \\
\hline EV difference & $0.00[0.00,0.00]$ & $0.00[0.00,0.00]$ \\
\hline Environment (Negative) & $-0.20[-0.53,0.13]$ & $-0.03[-0.42,0.36]$ \\
\hline Environment (Positive) & $0.07[-0.26,0.39]$ & $0.21[-0.18,0.59]$ \\
\hline \multicolumn{3}{|c|}{ Processing: Within-gamble transitions (4) } \\
\hline (Intercept) & $1.74[1.38,2.10]$ & $0.41[0.31,0.53]$ \\
\hline EV difference & $-0.01[-0.02,-0.01]$ & $0.00[0.00,0.00]$ \\
\hline Environment (Negative) & $-0.30[-0.79,0.26]$ & $-0.05[-0.21,0.10]$ \\
\hline Environment (Positive) & $-0.09[-0.59,0.41]$ & $0.05[-0.11,0.20]$ \\
\hline \multicolumn{3}{|c|}{ Processing: Gaze to payoff (5) } \\
\hline (Intercept) & $0.51[0.47,0.56]$ & $0.57[0.50,0.63]$ \\
\hline EV difference & $0.00[0.00,0.00]$ & $0.00[0.00,0.00]$ \\
\hline Environment (Negative) & $0.09[0.02,0.16]$ & $0.10[0.01,0.19]$ \\
\hline Environment (Positive) & $0.09[0.02,0.15]$ & $0.11[0.02,0.21]$ \\
\hline \multicolumn{3}{|c|}{ Choice: Higher EV pred. from process data (6) } \\
\hline (Intercept) & $0.41[0.11,0.72]$ & $-0.51[-0.78,-0.24]$ \\
\hline EV difference & $0.13[0.12,0.15]$ & $0.06[0.05,0.07]$ \\
\hline Response time & $-0.03[-0.06,0.00]$ & $0.47[0.25,0.69]$ \\
\hline AOIs inspected & $0.13[0.06,0.21]$ & $0.08[0.00,0.16]$ \\
\hline Transitions (within) & $0.01[-0.05,0.06]$ & $0.08[-0.04,0.21]$ \\
\hline Environment (Negative) & $-0.35[-0.60,-0.11]$ & $-0.20[-0.36,-0.03]$ \\
\hline Environment (Positive) & $-0.30[-0.55,-0.05]$ & $-0.14[-0.31,0.03]$ \\
\hline
\end{tabular}

Open Access This article is distributed under the terms of the Creative Commons Attribution 4.0 International License (http://creativecommons.org/licenses/by/4.0/), which permits unrestricted use, distribution, and reproduction in any medium, provided you give appropriate credit to the original author(s) and the source, provide a link to the Creative Commons license, and indicate if changes were made.

Publisher's Note Springer Nature remains neutral with regard to jurisdictional claims in published maps and institutional affiliations. 